
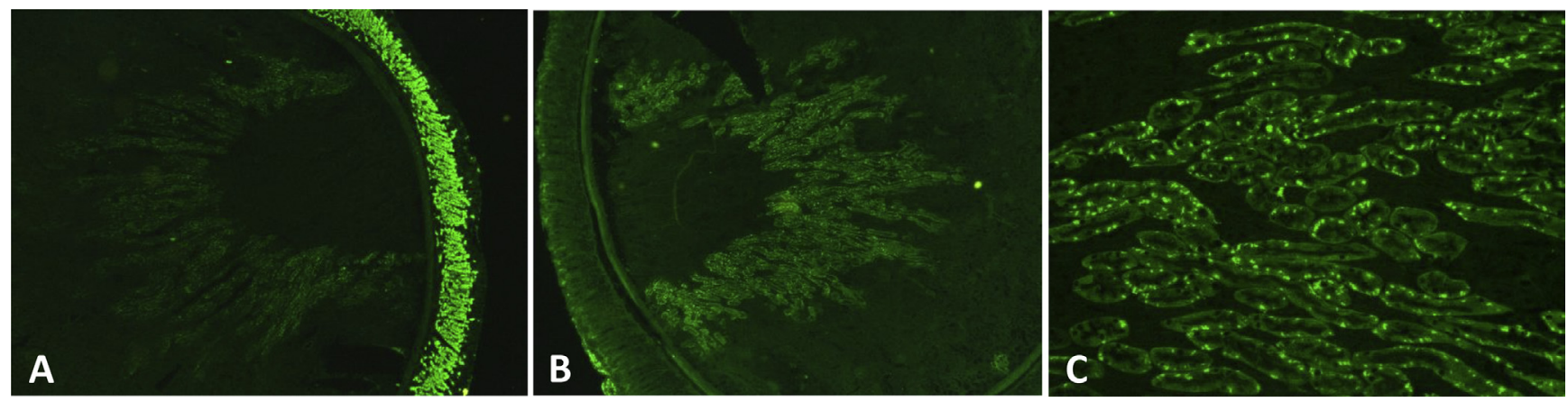

Fig. 1 Immunofluorescence staining on mouse kidney and stomach sections. (A) Strong staining of gastric parietal cells with faint HMGCR staining of distal kidney using subject's serum at 1 in 20 dilution. Gastric parietal tissue (far right), and thin muscularis layer, exterior to the kidney, where there is faint staining. (B) A CIEP confirmed anti-HMGCR positive serum at 1 in 20 dilution staining of mouse distal kidney. (C) A CIEP confirmed anti-HMGCR positive serum at 1 in 20 dilution staining of mouse distal kidney. (Nikon Image Navigator.)

all displayed to varying intensities the same distinctive immunofluorescence staining pattern on mouse kidney.

While this is an interesting observation it may not be suitable as a screening test for HMGCR antibodies. It is possible the presence of anti-mitochondrial or nuclear antibodies staining the entire tissue may mask this distinctive staining pattern. We also noted variability with the intensity of staining on different lots of tissue slides used, and slides from a different manufacturer did not give equivalent staining. Manufacturers' slide preparation process may contribute to the variability in staining. Further investigation is required to determine the suitability of mouse kidney tissue for routine anti-HMGCR immunofluorescence. However, as an incidental finding this unique staining pattern should prompt anti-HMGCR testing when observed during tissue autoantibody screening.

Conflicts of interest and sources of funding: The authors state that there are no conflicts of interest to disclose.

Paula Keating $^{1}$, Caroline Allan ${ }^{2}$, John O'Donnell ${ }^{1}$, Myfanwy Spellerberg ${ }^{1}$, Mark van Voorthuizen ${ }^{1}$

${ }^{1}$ Immunology Department, Canterbury Health Laboratories, Christchurch, New Zealand; ${ }^{2}$ Immunology, Lab Plus, Auckland City Hospital, Auckland, New Zealand

Contact Paula Keating.

E-mail: paula.keating@cdhb.health.nz

1. Christopher-Stine L, Casciola-Rosen LA, Hong G, et al. A novel autoantibody recognizing $200-\mathrm{kd}$ and $100-\mathrm{kd}$ proteins is associated with an immunemediated necrotizing myopathy. Arthritis Rheum 2010; 62: 2757-66.

2. Mammen AL, Chung T, Christopher-Stine L, et al. Autoantibodies against 3-hydroxy-3-methylglutaryl-coenzyme A reductase in patient with statin-associated autoimmune myopathy. Arthritis Rheum 2011; 63 $713-21$.

3. Keating P, Van Voorthuizen M, Liu J, et al. Response to: new immunoassays for anti-HMG-CoA reductase antibodies may lead to incorrect diagnosis in inflammatory myositis. Pathology 2018; 50: 588-90.

4. Droney L, Gillis D, Wong R. New immunoassays for anti-HMG-CoA reductase antibodies may lead to incorrect diagnosis in inflammatory myositis. Pathology 2017; 49: 638-9.

5. Alvarado-Cardenas M, Marin-Sanchez A, Martinez MA et al Statinassociated autoimmune myopathy: a distinct new IFL pattern can increase the rate of HMGCR antibody detection by clinical laboratories. Autoimmun Rev 2016; 15: 1161-6.

6. Zager RA, Johnson A. Renal cortical cholesterol accumulation is an integral component of the systemic stress response. Kidney Int 2001; 60 2299-310.

DOI: https://doi.org/10.1016/j.pathol.2020.10.020

\section{Mecillinam susceptibility in Victorian third generation cephalosporin-nonsusceptible Escherichia coli urinary isolates}

Sir,

Antimicrobial resistance has become an increasing challenge for clinicians managing urinary tract infections (UTIs). The worldwide emergence of the highly successful Escherichia coli ST131 clone is particularly problematic because it harbours multiple genes that confer resistance to commonly used antibiotics. Data from the Australian Group on Antimicrobial Resistance (AGAR) reported third generation cephalosporin resistance in $14.5 \%$ of $E$. coli causing bloodstream infections, for which the urinary tract was the most frequent source. Consecutive AGAR reports have also reported increasing rates of fluroquinolone and trimethoprim-sulfamethoxazole resistance. Comprehensive Australian data on third generation cephalosporin-nonsusceptible E. coli (3GCNS-EC) from urinary specimens are lacking. One Melbourne laboratory has reported $3 \mathrm{GCNS}-\mathrm{EC}$ rates of $5.7 \%$ from urinary isolates. ${ }^{2}$ Increasing rates of resistance to cephalosporins, ciprofloxacin and trimethoprim-sulfamethoxazole has resulted in limited treatment options for some infections. Fosfomycin, nitrofurantoin and pivmecillinam are frequently the only oral options available for 3GCNS-EC UTIs. ${ }^{3}$ Of these, nitrofurantoin is the only antibiotic listed on the Pharmaceutical Benefits Scheme, fosfomycin has been approved by the Therapeutic Goods Administration for use in females greater than 12 years of age, and pivmecillinam is available in Australia via the pharmaceutical Special Access Scheme. Fosfomycin is an attractive option for clinicians wishing to avoid the infrequently encountered adverse effects attributed to nitrofurantoin, but may be less effective. ${ }^{4}$

Pivmecillinam, an amidinopenicillin, is the orally active prodrug of mecillinam. Mecillinam is available in an intravenous formulation in a limited number of Scandinavian countries. In the laboratory pivmecillinam susceptibility is extrapolated from the results of antimicrobial susceptibility disk testing using mecillinam disks. Pivmecillinam demonstrates higher in vitro stability to $\beta$-lactamase hydrolysis compared to other penicillins and maintains good in vitro susceptibility against 3GCNS-EC. ${ }^{3,5}$ Furthermore, despite the use of pivmecillinam in Scandinavian countries for many years, widespread pivmecillinam resistance has not emerged, 
suggesting it has a higher barrier to resistance. ${ }^{6}$ Our study aimed to provide data on pivmecillinam susceptibility in E. coli urinary isolates from an Australian population. To our knowledge, these data are not currently available.

Our study was conducted at St Vincent's Pathology, Melbourne (St Vincent's Health Australia, Fitzroy, Australia). St Vincent's Pathology provides microbiology services to a public principal referral hospital, two private hospitals, two subacute care facilities and a range of general practice centres throughout Melbourne city and the surrounding suburbs. Approximately 5350 urinary specimens are received per month for microscopy and culture; approximately $65 \%$ of these are obtained from community patients. Between 1 July 2018 and 31 December 2019, 98 consecutive, first isolate, non-duplicate $3 \mathrm{GCNS}-\mathrm{EC}$ [ceftriaxone minimum inhibitory concentration (MIC) $>1.0 \mu \mathrm{g} / \mathrm{mL}$ or ceftazidime MIC $>4.0 \mu \mathrm{g} / \mathrm{mL}]$ and 101 consecutive, first isolate, nonduplicate third generation cephalosporin-susceptible isolates identified from urinary specimens with microscopy consistent with urinary tract infection were included in this study.

The isolate identity was determined using matrix assisted laser desorption ionisation-time of fight mass spectrometry (MALDI-TOF MS) (Bruker Daltonik, Germany). Antimicrobial susceptibility testing was performed using VITEK2 (bioMérieux, France) with an AST-N246 card, from which susceptibility to ceftriaxone, ceftazidime, meropenem, ciprofloxacin, nitrofurantoin, trimethoprim and trimethoprimsulfamethoxazole was obtained. Categorical interpretation of VITEK2 MICs were performed using Clinical and Laboratory Standards Institute (CLSI) Guidelines (30th edition, 2020). Pivmecillinam susceptibility was determined using disk diffusion in accordance with CLSI and European Committee on Antimicrobial Susceptibility Testing (EUCAST) guidelines. In brief, a $10 \mu \mathrm{g}$ mecillinam disk (Oxoid, Australia) was added to a lawn culture of the test isolate inoculated onto Mueller-Hinton E Agar (bioMérieux). The isolate was incubated overnight in air at $35-37^{\circ} \mathrm{C}$. Inhibition zones were read using digital calipers and checked by a second operator. Categorical determination of susceptibility was determined using CLSI and EUCAST breakpoints for zone diameters. ${ }^{7,8}$ Likewise fosfomycin susceptibility was performed by applying a $200 \mu \mathrm{g}$ fosfomycin disk (Oxoid) supplemented with $50 \mu \mathrm{g}$ of glucose-6-phosphate to a lawn of test isolate on Mueller-Hinton E Agar. The isolate was incubated for 18-24 hours in air at $35-37^{\circ} \mathrm{C}$. Inhibition zones were read using digital calipers and checked by a second operator. Categorical determination of susceptibility was determined using CLSI and EUCAST breakpoints for zone diameters. ${ }^{7,8}$ For fosfomycin inhibition zone determination, individual colony ingrowth was ignored as per reading instructions.

All 101 3GS-EC and all 98 3GCNS-EC underwent genotypic analysis. For genotypic analysis, isolates were stored in glycerol at $4{ }^{\circ} \mathrm{C}$ and subcultured onto trypticase-soy agar with sheep blood (Oxoid) for batch testing. For each isolate, a single colony was inoculated into $2 \mathrm{~mL}$ of buffer and vortexed for $10 \mathrm{~s}$ to achieve an even distribution. Isolate suspensions were then analysed for the presence of resistance genes using the 16-well ESBL PCR assay (AusDiagnostics, Australia), which includes testing for the following $\beta$-lactamase resistance genes: TEM, SHV, panTEM, VEB, DHA-1, CYM1, CTX-M group 1, CTX-M group 9, VIM, pan-IMP, KPC, OXA-1 and OXA-48 like. Isolate suspensions were tested in batches of 23 with a negative control run with every batch. A positive internal control is included in the AusDiagnostics 16-well ESBL PCR assay. VITEK2 susceptibility data were directly downloaded and imported into Excel (Microsoft, USA). Likewise, PCR results were downloaded from the AusDiagnostics software and imported into Excel. Pivmecillinam and fosfomycin susceptibility results were manually entered into Excel with a second investigator checking transcription.

Using CLSI interpretive guidelines, 170 (85.4\%) isolates were susceptible to pivmecillinam, 197 (98.9\%) isolates were susceptible to fosfomycin and 194 (97.5\%) isolates were susceptible to nitrofurantoin (Table 1). Overall rates of susceptibility to pivmecillinam, fosfomycin and nitrofurantoin did not substantially differ amongst 3GCNS-EC and susceptible E. coli isolates. Pan-TEM, CTX-M group 9 and CTX-M group 1 were the most frequent $\beta$-lactamase genes detected in the 3GCNS-EC isolates (Fig. 1). Ambler class $C$ $\beta$-lactamases (CMY and DHA) were less frequently observed. No carbapenemase-producing genes were detected. Pan-TEM, pan-SHV and VEB genes were detected in some third generation cephalosporin-susceptible isolates (Fig. 1).

This small study suggests that pivmecillinam presents a good therapeutic option for Australian clinicians, with a susceptibility in all E. coli isolates of $85.4 \%$ and a susceptibility in 3GCNS-EC of $87.8 \%$. These susceptibility rates are slightly lower than those observed in studies from Germany (97.4\%) and the United Kingdom (96\%) where similar methods of antimicrobial susceptibility testing are utilised. We did detect a significant amount of TEM and some SHV $\beta$ lactamase genes in our 3GCNS-EC isolates and these enzymes have greater activity against pivmecillinam. Further data on $\beta$-lactamase frequencies and pivmecillinam susceptibility in the Australian and global context would be required to definitively conclude that this is the explanation for our observed lower pivmecillinam susceptibility rates. CTX-M was the most commonly detected $\beta$-lactamase in our 3GCNS-EC isolates which is consistent with data from other Australian studies.

There are several situations where clinicians may be reluctant to use nitrofurantoin including in elderly patients and in patients with renal impairment. Long term use may be associated with the rare adverse drug reaction of pulmonary

Table 1 Pivmecillinam, fosfomycin and nitrofurantoin susceptibilities in third generation cephalosporin-susceptible and third generation cephalosporin-nonsusceptible E. coli isolates from urine

\begin{tabular}{|c|c|c|c|c|}
\hline & \multicolumn{2}{|c|}{$\begin{array}{l}\text { Third generation } \\
\text { cephalosporin- } \\
\text { susceptible } \\
\text { E. coli } \\
(n=101)\end{array}$} & \multicolumn{2}{|c|}{$\begin{array}{l}\text { Third generation } \\
\text { cephalosporin- } \\
\text { nonsusceptible } \\
\text { E. coli } \\
(n=98)\end{array}$} \\
\hline & CLSI & EUCAST & CLSI & EUCAST \\
\hline \multicolumn{5}{|l|}{ Pivmecillinam } \\
\hline Susceptible & 84 & 84 & 86 & 86 \\
\hline Intermediate & 6 & - & 6 & - \\
\hline Resistant & 11 & 17 & 6 & 12 \\
\hline \multicolumn{5}{|l|}{ Fosfomycin } \\
\hline Susceptible & 101 & 97 & 96 & 88 \\
\hline Intermediate & 0 & - & 1 & - \\
\hline Resistant & 0 & 4 & 1 & 10 \\
\hline \multicolumn{5}{|l|}{ Nitrofurantoin } \\
\hline Susceptible & 99 & - & 95 & - \\
\hline Resistant & 2 & - & 3 & - \\
\hline
\end{tabular}




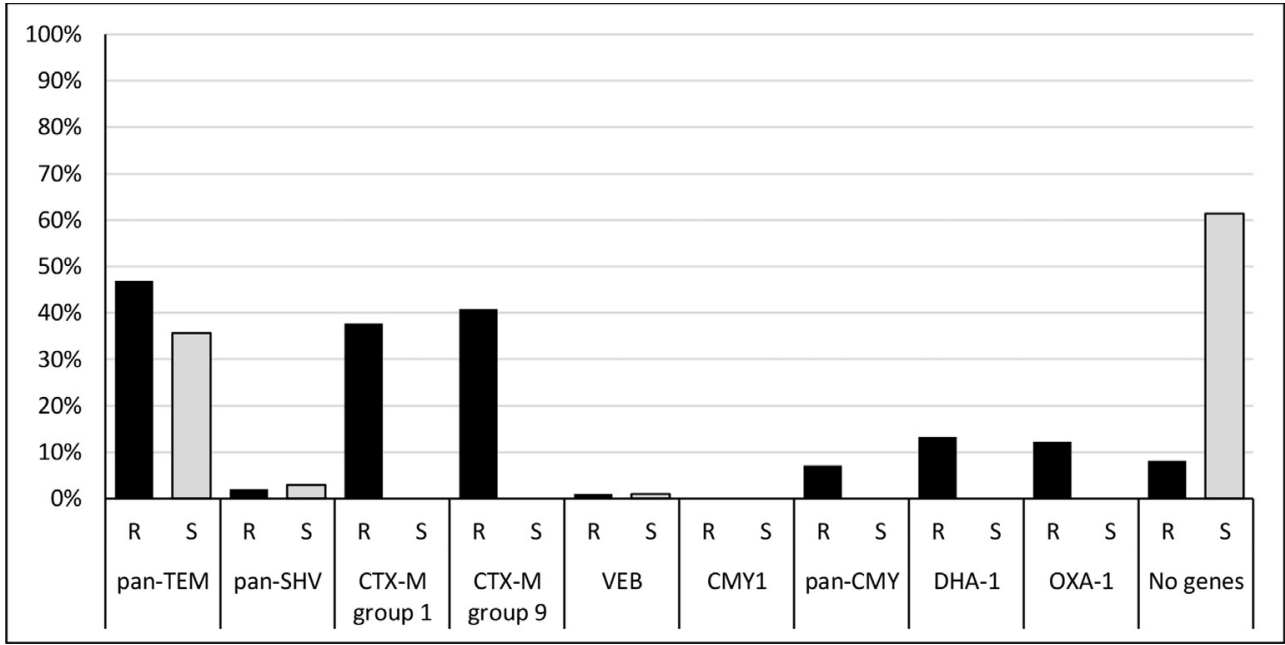

Fig. 1 Resistance genes detected in third generation cephalosporin nonsusceptible (R, black bars) and susceptible (S, grey bars) E. coli isolates from urinary specimens.

fibrosis. ${ }^{10}$ Fosfomycin offers an additional option but gastrointestinal side effects are common and the development of resistance is commonly observed. ${ }^{1}$

Pivmecillinam has a low prevalence of gastrointestinal side effects, can be safely used in renal impairment and has a high barrier to resistance. ${ }^{6,12}$ Australian laboratories should consider testing for mecillinam susceptibilitiy in E. coli isolates from patients with recurrent, problematic multidrug resistant urinary tract infections.

Conflicts of interest and sources of funding: The authors state that there are no conflicts of interest to disclose.

\section{Maxwell Olenski ${ }^{1}$, Darren Jardine ${ }^{2}$, Yves S. Poy Lorenzo $^{1}$, Amy Crowe ${ }^{1,2}$}

${ }^{1}$ St Vincent's Hospital Melbourne, Melbourne, Vic Australia; ${ }^{2}$ St Vincent's Pathology Melbourne, Melbourne, Vic, Australia

Contact Dr Amy Crowe.

E-mail: amy.crowe@svha.org.au

1. Australian Group on Antimicrobial Resistance. Sepsis Outcome Programs: Gram-Negative Sepsis Outcome Program 2018 Report. Cited 20 Feb 2020. https://agargroup.org.au/agar-surveys\#Gram-NegativeBacteria

2. Chua KYL, Stewardson AJ. Individual and community predictors of urinary ceftriaxone-resistant Escherichia coli isolates, Victoria, Australia. Antimicrob Resist Infect Control 2019; 8: 36.

3. Raja NS. Oral treatment options for patients with urinary tract infections caused by extended spectrum ßeta-lactamase (ESBL) producing Enterobacteriaceae. J Infect Publ Health 2019; 12: 843-6.

4. Huttner A, Kowalczyk A, Tujeman A, et al. Effect of 5-day nitrofurantoin vs single dose fosfomycin on clinical resolution of uncomplicated lower urinary tract infection in women: a randomized clinical trial. JAMA 2018; 319: 1781-9.

5. Dewar S, Reed LC, Koerner RJ. Emerging role of pivmecillinam in the treatment of urinary tract infection in the context of multi-drug resistan bacteria. J Antimicrob Chemother 2014; 69: 303-8.

6. Hoiby N. Ecological antibiotic policy. J Antimicrob Chemother 2000; 46: 59-62.

7. Clinical and Laboratory Standards Institute (CLSI). Performance Standards for Antimicrobial Susceptibility Testing. 30th ed. CLSI document M100. Wayne, PA: CLSI, 2020.

8. European Committee on Antimicrobial Susceptibility Testing. Breakpoint tables for interpretation of MICs and zone diameters v10. Cited 20 Feb 2020. https://www.eucast.org/clinical_breakpoints/
9. Harris PNA, Zakour NLB, Roberts LW, et al. Whole genome analysis of cephalosporin-resistant Escherichia coli from bloodstream infections in Australia, New Zealand and Singapore: high prevalence of CMY-2 producers and ST131 carrying bla ${ }_{\text {CTX-M-15 and bla }}$ CTX-M-27. J Antimicrob Chemother 2018; 73: 634-42.

10. Grayson ML, Whitby M. Nitrofurans: nitrofurazone, furazolidone and nitrofurantoin. In: Grayson ML, Crowe SM, editors, Kucers' the Use of Antibiotics. 6th ed. London: Hodder Arnold, 2010; 1195-204.

11. Wenzeler E, Bleasdale S, Sikka M, et al. Phase 1 study to evaluate the pharmacokinetics, safety and tolerability of two dosing regimens of oral fosfomycin tromethamine in health adult participants 2018. Antimicrob Agents Chemother 2018; 62: e00464-18.

12. Ferry SA, Holm SE, Stenlund $\mathrm{H}$, et al. Clinical and bacteriological outcome of different doses and duration of pivmecillinam compared with placebo therapy in uncomplicated lower urinary tract infection in women: the LUTIW project. Scand J Prim Health Care 2007; 25 $49-57$.

DOI: https://doi.org/10.1016/j.pathol.2020.10.016

\section{Discrepancy between VITEK2 and Etest aminoglycoside susceptibility testing for multidrug- resistant Acinetobacter baumannii}

Sir,

Acinetobacter baumannii is an important Gram-negative organism responsible for various nosocomial infections and is frequently multidrug-resistant. We report a case of carbapenem-resistant Acinetobacter baumannii infection with discrepant aminoglycoside susceptibility testing results. Whole genome sequencing identified a $16 \mathrm{~S}$ methylase gene mediating aminoglycoside resistance and OXA-23 carbapenemase.

A 24-year-old previously well male was transferred to an Australian tertiary hospital from Thailand following a motorbike accident one month prior in Vietnam. He sustained multiple intracranial, maxillofacial and orthopaedic injuries requiring bilateral decompressive craniectomies, tracheostomy placement and prolonged intensive care admission. His orthopaedic injuries included compound left femoral, patella and tibial fractures, requiring open reduction and fixation, 